\title{
Strong biases in retrieved atmospheric composition caused by day-night chemical heterogeneities
}

\author{
William Pluriel ${ }^{1}$, Tiziano Zingales ${ }^{1}$, Jérémy Leconte ${ }^{1}$ and Vivien Parmentier ${ }^{2}$ \\ ${ }^{1}$ Laboratoire d'astrophysique de Bordeaux, Univ. Bordeaux, CNRS, B18N, allée Geoffroy Saint-Hilaire, 33615 Pessac, France \\ e-mail: william.pluriel@u-bordeaux.fr \\ 2 Department of Physics, Oxford University, OX1 2JD, UK \\ e-mail: vivien.parmentier@physics.ox.ac.uk
}

Received 7 February 2020 / Accepted 12 March 2020

\begin{abstract}
Most planets currently amenable to transit spectroscopy are close enough to their host stars to exhibit a relatively strong day to night temperature gradient. For hot planets this leads to a chemical composition dichotomy between the two hemispheres. In the extreme case of ultra-hot Jupiters, some species, such as molecular hydrogen and water, are strongly dissociated on the day side while others, such as carbon monoxide, are not. However, most current retrieval algorithms rely on 1D forward models that are unable to reproduce this effect. We thus investigate how the 3D structure of the atmosphere biases the abundances retrieved using commonly used algorithms. We study the case of Wasp-121b as a prototypical ultra-hot Jupiter. We use the simulations of this planet performed with the Substellar and Planetary Atmospheric Radiation and Circulation global climate model and generate transmission spectra that fully account for the 3D structure of the atmosphere with Pytmosph3R. These spectra are then analyzed using the TauREx retrieval code. We find that the ultra-hot Jupiter transmission spectra exhibit muted $\mathrm{H}_{2} \mathrm{O}$ features that originate on the night side where the temperature, hence the scale-height, is smaller than on the day side. However, the spectral features of molecules present on the day side are boosted by both its high temperature and low mean molecular weight. As a result, the retrieved parameters are strongly biased compared to the ground truth. In particular the $[\mathrm{CO}] /\left[\mathrm{H}_{2} \mathrm{O}\right]$ is overestimated by one to three orders of magnitude. This must be kept in mind when using the retrieval analysis to infer the $\mathrm{C} / \mathrm{O}$ of a planet's atmosphere. We also discuss whether indicators can allow us to infer the 3D structure of an observed atmosphere. Finally, we show that Wide Field Camera 3 from Hubble Space Telescope transmission data of Wasp-121b are compatible with the day-night thermal and compositional dichotomy predicted by models.
\end{abstract}

Key words. planets and satellites: atmospheres - radiative transfer - techniques: spectroscopic - methods: numerical

\section{Introduction}

Since the discovery of the first exoplanets, observations have shown a great diversity of objects, from Earth-like planets to ultra-hot Jupiters (UHJ). Orbiting very close to their star, UHJs can reach atmospheric temperatures high enough to trigger the thermal dissociation of some chemical species, such as $\mathrm{H}_{2} \mathrm{O}$ and $\mathrm{H}_{2}$ (Lodders \& Fegley 2002; Visscher et al. 2006, 2010). They thus offer the opportunity to study the chemistry and physics of planetary atmospheres under extreme conditions, for which we have no equivalent in the Solar System. These interesting objects will be prime targets for a new generation of space missions like the James Webb Space Telescope (JWST; Beichman et al. 2014) and Atmospheric Remote-sensing Infrared Exoplanet Large-survey (ARIEL; Tinetti et al. 2018).

Currently only a few UHJs have been discovered and studied using both transit and eclipse spectroscopy (Wright et al. 2012; Haynes et al. 2015; Sheppard et al. 2017; Evans et al. 2017; Kreidberg et al. 2018). However, the analysis of UHJs is not simple, due to their complex chemical composition and dynamics. Bayesian retrieval procedures being computationally intensive, it is necessary to make strong assumptions to speed-up the atmospheric forward model. However, a set of assumptions that is too simplistic can lead to strongly biased interpretations where the retrieved abundances are much higher than expected. Evans et al. (2017), for example, used transit data (that suggested a supersolar FeH abundance) to fit Wide Field Camera 3 from Hubble
Space Telescope (HST-WFC3) data of Wasp-121b and to explain the reduced water shape around $1.3 \mu \mathrm{m}$. Parmentier et al. (2018) later suggest that this could be due to the presence of $\mathrm{CaTiO}_{3}$ in a partially cloudy atmosphere.

In the present study, we investigate whether the variations in composition inside the atmosphere of UHJs may affect transmission spectroscopy as severely as emission spectroscopy. These tidally locked planets present a strong day-night contrast both in temperature (Sudarsky et al. 2000; Bell \& Cowan 2018; Arcangeli et al. 2018) and in chemical heterogeneities due to the thermal dissociation of certain species such as $\mathrm{H}_{2} \mathrm{O}$ and $\mathrm{H}_{2}$ (Parmentier et al. 2018; Marley et al. 2017). As the thermal dissociation of the species is strongly linked to the temperature, the thermal day-night dichotomy entails a chemical dichotomy, with a day side devoid of water above about 100 mbar and a night side where water is present everywhere. However, other species (like $\mathrm{CO}$ ) require higher temperatures to dissociate (Lodders \& Fegley 2002) and are expected to remain constant in the atmosphere, which would change the apparent $[\mathrm{CO}] /\left[\mathrm{H}_{2} \mathrm{O}\right]$ ratio. Caldas et al. (2019) developed a fully 3D model that allows us to generate a transmission spectrum which considers the 3D structure of the atmosphere. They show that the light that goes through the planetary atmosphere carries the information of a portion of the atmospheres that significantly extend around the limb. They highlight that the rays of light go through the day side first and then to the night side, implying that strong $3 \mathrm{D}$ effects on the transmission spectrum across the limb occur when the effects 
along the limb are negligible. Hence, 1D transmission models cannot fully take into account the chemical heterogeneities described above, thus the need to use 3D transmission models.

Caldas et al. (2019) highlighted systematic biases on retrieved temperatures using a 1D retrieval model TauREx (Waldmann et al. 2015a,b). However, this earlier study only looked at atmospheres with a homogeneous composition to focus on thermal effects. To that purpose, we focus on Wasp-121b (Evans et al. 2016, 2017; Parmentier et al. 2018) as a prototype for UHJs. The complexity of the UHJ atmospheres describes in our global climate model (GCM ) model suggest that we need a more complex framework to analyze the transmission spectra of those particular planets. Thus, we will need fully 3D forward models to simulate realistic transmission spectra to better fit transit observations of UHJs. Hence, 1D retrieval models such as TauREx are probably biased in their analysis.

The structure of the paper is as follows. We describe the observational chain used to simulate JWST observations in Sect. 2. Then in Sect. 3 we explain the numerical experiments done to unravel the biases starting from a very simple parametric modeling of Wasp-121b to a more elaborate GCM model. Moreover, we explain how thermal dissociation induces strong heterogeneities of composition between the day and night sides of the planet. We describe our retrieval results in Sect. 4. Finally, we highlight the main conclusions of our study, and we discuss the limitations of our method and our models in Sect. 5.

\section{Presentation of our spectra generation and retrieval framework}

\subsection{SPARC/MITgcm global circulation model}

We use the Substellar and Planetary Atmospheric Radiation and Circulation SPARC/MITgcm global circulation model (Showman et al. 2009) to model the atmosphere of WASP-121b. The model solves the primitive equations on a cubic-sphere grid. It has been successfully applied to a wide range of hot Jupiters (Showman et al. 2009; Kataria et al. 2015; Lewis et al. 2017; Parmentier et al. 2013, 2016) and has recently been used to study a few ultra-hot Jupiters in detail (Kreidberg et al. 2018; Parmentier et al. 2018; Arcangeli et al. 2019).

The model used here is exactly the same as described in Parmentier et al. (2018). Our pressure ranges from 200 bar to $2 \mu$ bar over 53 levels, We use a horizontal resolution of C32, equivalent to an approximate resolution of 128 cells in longitude and 64 in latitude. The radiative transfer is handled with a two-stream radiation scheme (Marley \& McKay 1999), with the opacities treated using eight correlated-k coefficients (Goody \& Yung 1989) within each of our 11 wavelength bins (Kataria et al. 2013).

Chemical equilibrium is assumed when calculating the opacities, meaning that thermal dissociation of molecules, including water and hydrogen, is naturally taken into account. However, for practical purposes we assume that the mean molecular weight and the heat capacity are constant throughout the atmosphere. The change in mean molecular weight is explored a posteriori when projecting the global circulation model (GCM) thermal structure in the Pytmosph3R grid (see Sect. 2.2). This model does not consider the impact of $\mathrm{H}_{2}$ recombination in the atmosphere, which can have a non-negligible effect on the physics and the dynamics (Tan \& Komacek 2019; see Sect. 3.1 for further details).

Figures 1 and 2 show the temperature maps generated by the GCM which are structured in three main parts:
1. A quasi-isothermal annulus around $2500 \mathrm{~K}$ from the surface pressure to approximately a pressure of $0.1 \mathrm{bar}$. This is in the deep atmosphere, at high pressure, and thus the redistribution of energy is very efficient due to jet stream.

2. A cold night side above this annulus, where the temperature gradient decreases with altitude going from 1000 to $500 \mathrm{~K}$ at whole latitudes.

3. A very hot day side where the temperature gradient decreases with altitude from 3500 to $3000 \mathrm{~K}$ at whole latitudes. These high temperatures enlarge the scale height implying a strong asymmetry in altitude between the day and the night side.

The transition between the day and the night side is very sharp, with a quasi-isothermal terminator around $2200 \mathrm{~K}$. We used this global thermal structure in our study to build an idealize case of Wasp-121b (Figs. 3-4), which is explained in Sect 3.

As introduced before, the temperatures are high enough to allow dissociation of species, especially water. We calculate the abundance of $\mathrm{H}_{2} \mathrm{O}$ in the GCM simulations using the analytical equations provided by Parmentier et al. (2018) and we plot the abundances maps of $\mathrm{H}_{2} \mathrm{O}$ in Figs. 1 and 2. We can see on the equatorial and polar maps that the water abundance is linked to the temperature since there is a total absence of water deep in the atmosphere on the day side, while the water abundance reaches solar abundance on the night side. The pressure dependence of water dissociation appears in the limb map, which is quasi-isothermal, since the water abundance reaches the solar abundance in the surface pressure then decreases to less than $10^{-12}$ at the top of the atmosphere (see Fig. 5).

\subsection{Generation of transmission spectra with Pytmosph3R}

Transmission spectra are computed using Pytmosph3R as described in Caldas et al. (2019). Pytmosph3R is designed to simulate transmission spectra based on any 3D atmospheric structure, including outputs of a GCM. It produces transmittance maps at any wavelength that can later be spatially integrated to yield the transmission spectrum. The code can account for molecular opacities using the correlated-k method ( Fu \& Liou 1992) or monochromatic cross sections calculated by ExoMol (Yurchenko et al. 2011, 2014; Tennyson \& Yurchenko 2012; Barton et al. 2013, 2014). In this study, we only use the latter to ensure a complete compatibility with the TauREx retrieval code (Waldmann et al. 2015b,a). Unless stated otherwise, our simulations only include $\mathrm{H}_{2}, \mathrm{He}, \mathrm{H}_{2} \mathrm{O}$, and $\mathrm{CO}$. We also take into account $\mathrm{H}_{2}-\mathrm{H}_{2}$ and $\mathrm{He}-\mathrm{H}_{2}$ continua and the atmospheric scattering. To simplify the analysis, we did not include $\mathrm{TiO}$ and $\mathrm{VO}$ to focus on the molecules above.

The abundances of the species in the atmosphere are calculated based on the temperature given by the GCM simulation and following Parmentier et al. (2018) when dissociation occurs as described in Sect 2.1. The molecular weight then derives from these abundances. The gravity and the atmospheric scale height vary with the altitude from the planetary surface, i.e., the radius of the planet at a pressure of 10 bar, as described in Caldas et al. (2019), and are defined, respectively, in Eqs. (1) and (2):

$$
\begin{aligned}
& g(z)=g_{0} \frac{1}{\left(1+\frac{z}{R_{p}}\right)^{2}} \\
& H(z)=\frac{k T(z)}{\mu(z) g(z)} .
\end{aligned}
$$

Here $g_{0}$ is the surface gravity, $R_{\mathrm{p}}$ is the planetary radius, $T$ is the temperature, and $\mu$ is the mean molecular mass. 
W. Pluriel et al.: Strong biases in retrieved atmospheric composition caused by strong day-night chemical heterogeneities
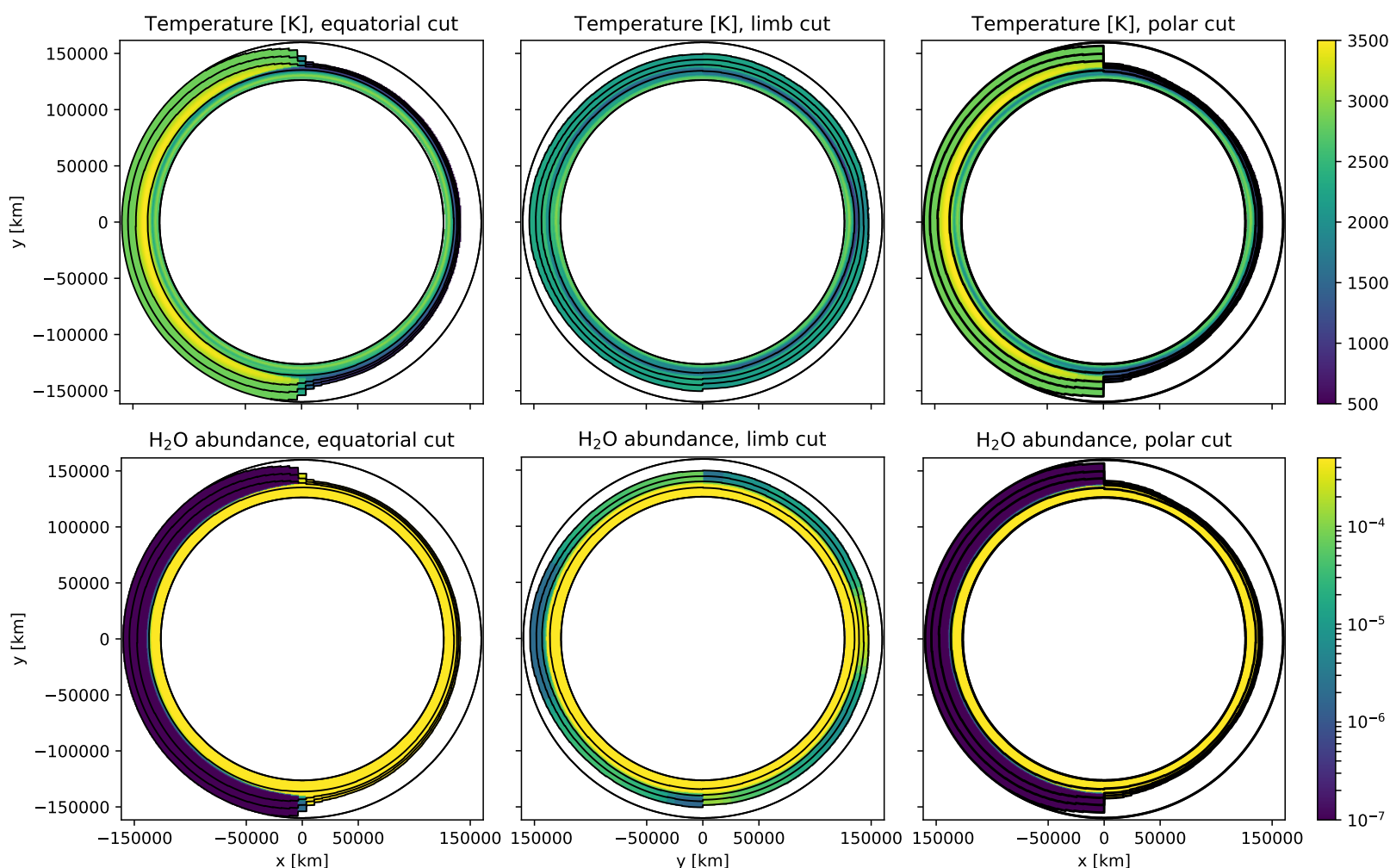

Fig. 1. GCM simulation of Wasp-121b (Parmentier et al. 2018) without $\mathrm{H}_{2}$ dissociation. Temperature (top) and water abundance (bottom) for the equatorial cut (left), limb cut (middle), and pole cut (right). From the center outward, the five solid lines are respectively the $1,434 \times 10^{7}, 10^{3}, 1$, $10^{-2}$, and $10^{-4} \mathrm{~Pa}$ pressure levels. The color-coding for the water abundance maps goes from $5 \times 10^{-4}$ to $10^{-7}$. The radius of the planet and the atmosphere are shown to scale.
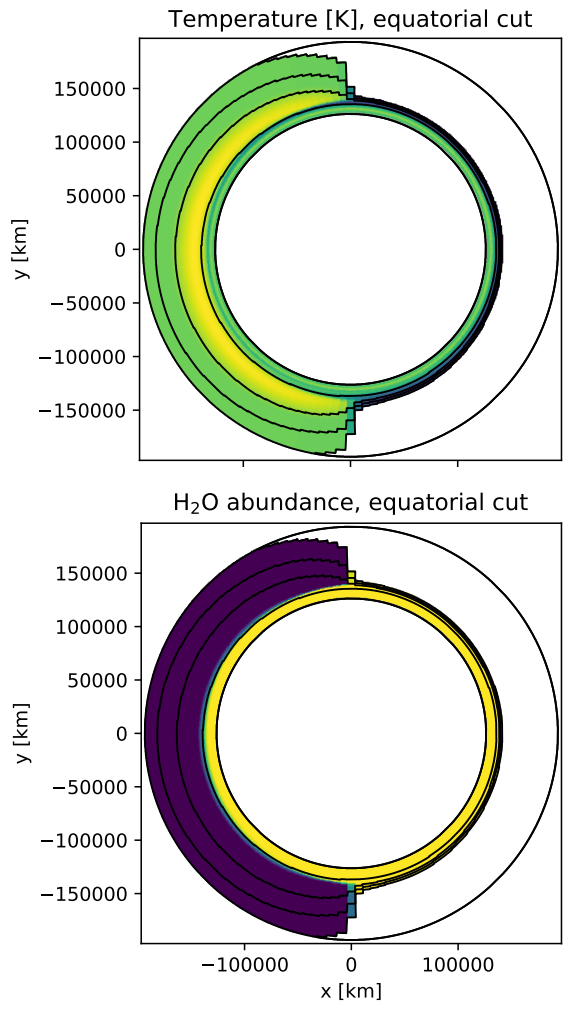

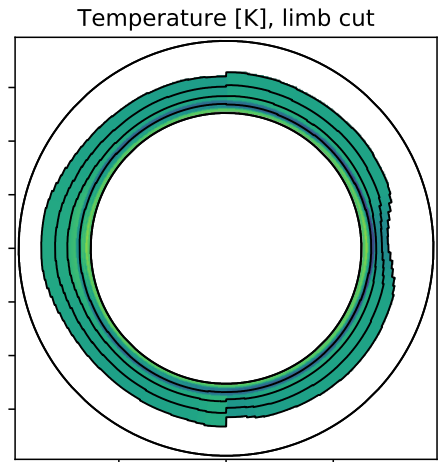

$\mathrm{H}_{2} \mathrm{O}$ abundance, limb cut

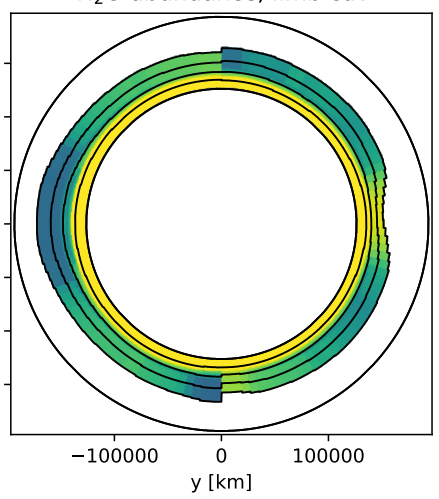

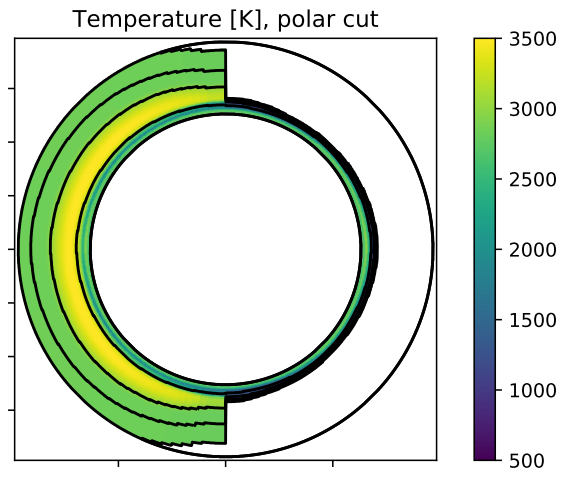

$\mathrm{H}_{2} \mathrm{O}$ abundance, polar cut

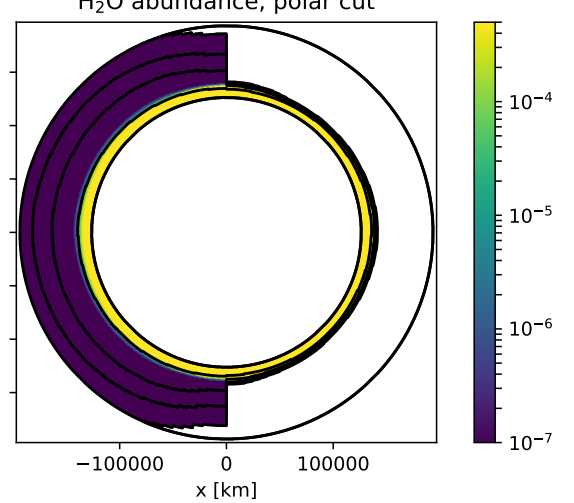

Fig. 2. Same as in Fig. 1, but taking into account $\mathrm{H}_{2}$ dissociation. We clearly see that the dissociation of $\mathrm{H}_{2}$ mostly affects the day side of the atmosphere, which further increases the dichotomy between the day and night sides. 

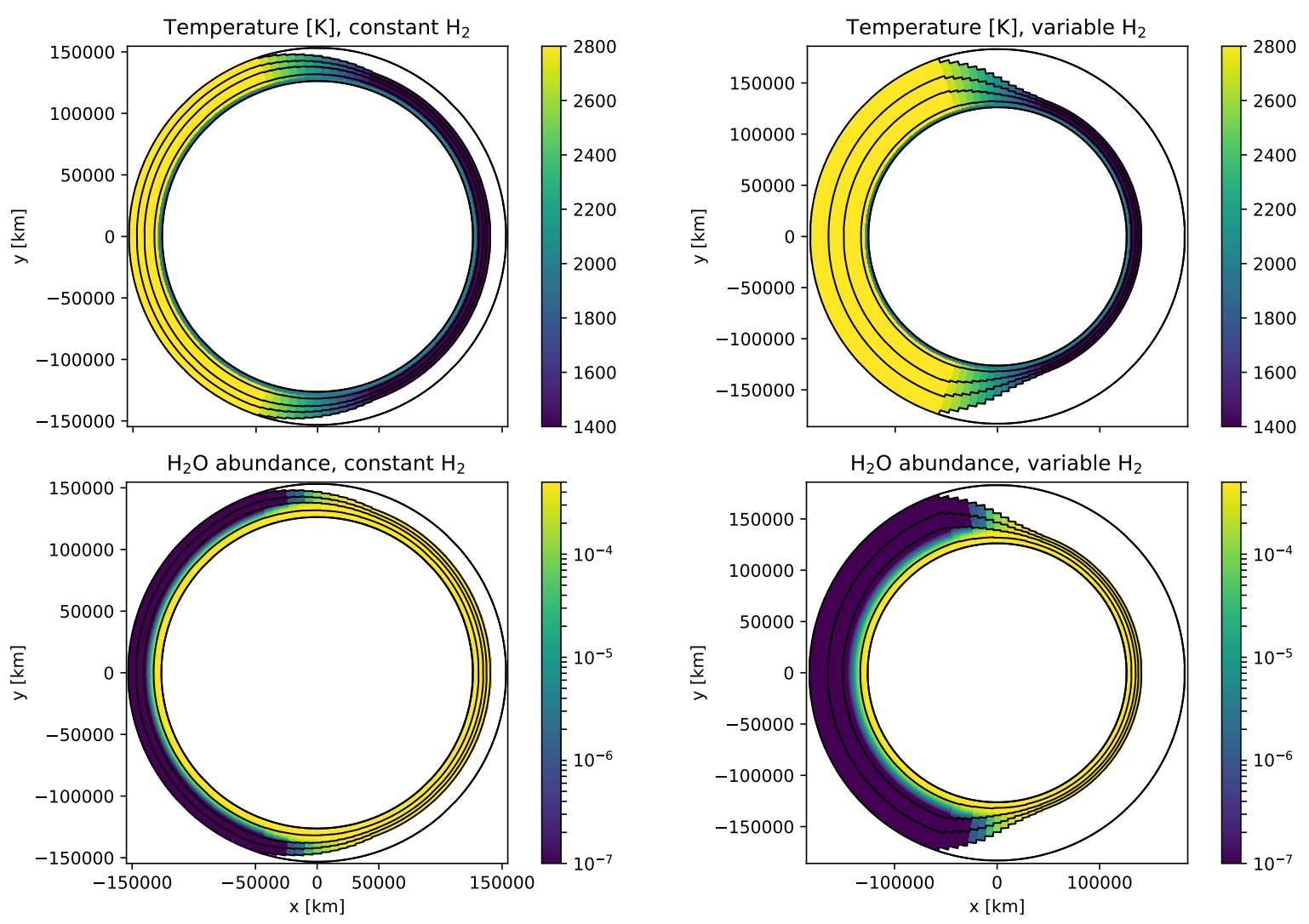

Fig. 3. Atmospheric structure of the symmetric idealized case, $\nabla^{-} T$, assuming an absence of and the presence of $\mathrm{H}_{2}$ dissociation (left and right, respectively). We show the temperature map (top) and the water abundance map (bottom). The temperature gradient goes from 1400 to $2800 \mathrm{~K}$. The transmission angle is $20^{\circ}$ around the terminator line and has a $2500 \mathrm{~K}$ ring at $P=0.13$ bar from the surface pressure. From the center outward, the five solid lines are, respectively, the $10^{6}, 10^{3}, 1,10^{-2}$, and $10^{-4}$ Pa pressure levels. The color-coding for the water abundance maps goes from $5 \times 10^{-4}$ to $10^{-7}$. The radius of the planet and the atmosphere are shown to scale.

We define the volume mixing ratio (VMR) as

$x_{\mathrm{i}}=\frac{N_{\mathrm{i}}}{\sum N_{\mathrm{i}}}$,

where $N_{\mathrm{i}}$ is the molecular number density in molecules per volume unit. We also assume that all the other species are present in the atmosphere as trace gases, so we can write the number density of atomic hydrogen as

$N_{\mathrm{H}}=2\left(N_{\mathrm{H}_{2}}^{0}-N_{\mathrm{H}_{2}}\right)$,

where $N_{\mathrm{H}_{2}}^{0}$ is the molecular number density when no dissociation occur (e.g. deep in the atmosphere; see Table 1).

We ran Pytmosph3R at $R=10000$ resolution, with $R=\frac{\lambda}{\Delta \lambda}$, in the spectral range from 0.6 to $10 \mu \mathrm{m}$. The spectra were then binned down to a resolution of $R=100$ for the retrievals. We used this low spectral resolution for several reasons:

1. We simulate JWST observations from 0.6 to $10 \mu \mathrm{m}$ using the low resolution prism mod provided by Near-Infrared Spectrograph (NIRSpec) and Mid Infra-Red Instrument (MIRI) instruments (Stevenson et al. 2016). This does not mean that using the prism configuration is the best observational strategy for such a bright target, but it provides us with a uniformly sampled spectrum that does not arbitrarily put more weight in the retrieval for some spectral regions.

2. We compared TauREx with the fully homogeneous case planet, as in Caldas et al. (2019), and we saw that the optimum resolution for the best accuracy in the retrieval is the resolution $R=100$.

3 . There are only two absorbing species in our atmosphere $\left(\mathrm{H}_{2} \mathrm{O}\right.$ and $\left.\mathrm{CO}\right)$ and we do not need to resolve specific lines, but large features in large spectral bands.

In all our simulations we used the planetary and stellar parameters shown in Table 2. In the part of the atmosphere where $\mathrm{H}_{2}$ does not dissociate, we have a fixed $\frac{\mathrm{He}}{\mathrm{H}_{2}}$ ratio at 0.25885 . Then we compute the volume mixing ratios of $\mathrm{H}$ and $\mathrm{He}$ by combining Eqs. (3) and (4) where $\mathrm{H}_{2}$ dissociates.

Since the overall flux is dominated by the star, we assume that the spectral noise is dominated by the stellar photon noise, defined as

$N_{\text {phot }}=\frac{\pi \tau \Delta t}{h c}\left(\frac{R_{*} D}{2 d}\right)^{2} \int_{\lambda_{1}}^{\lambda_{2}} B\left(\lambda, T_{*}\right) \lambda \mathrm{d} \lambda$,

where $\lambda_{1}$ and $\lambda_{2}$ are the limiting wavelengths of the bin considered, $d$ is the distance of the star (270 pc for WASP-121), and $R_{*}$ and $T_{*}$ are respectively the stellar radius and the effective temperature. The parameters $D, \tau$, and $\Delta t$ are respectively the telescope diameter, the system throughput, and the integration time, whose values have been fixed for JWST according to Cowan et al. (2015).

Using Eq. (5), the uncertainty varies from 10 to $50 \mathrm{ppm}$ between 1 and $10 \mu \mathrm{m}$, assuming a single WASP-121b transit. Since systematics may prevent us from reaching a 10ppms precision with JWST, wherever the shot noise was lower than $30 \mathrm{ppms}$ we assumed a floor noise of 30ppms through the whole 
W. Pluriel et al.: Strong biases in retrieved atmospheric composition caused by strong day-night chemical heterogeneities
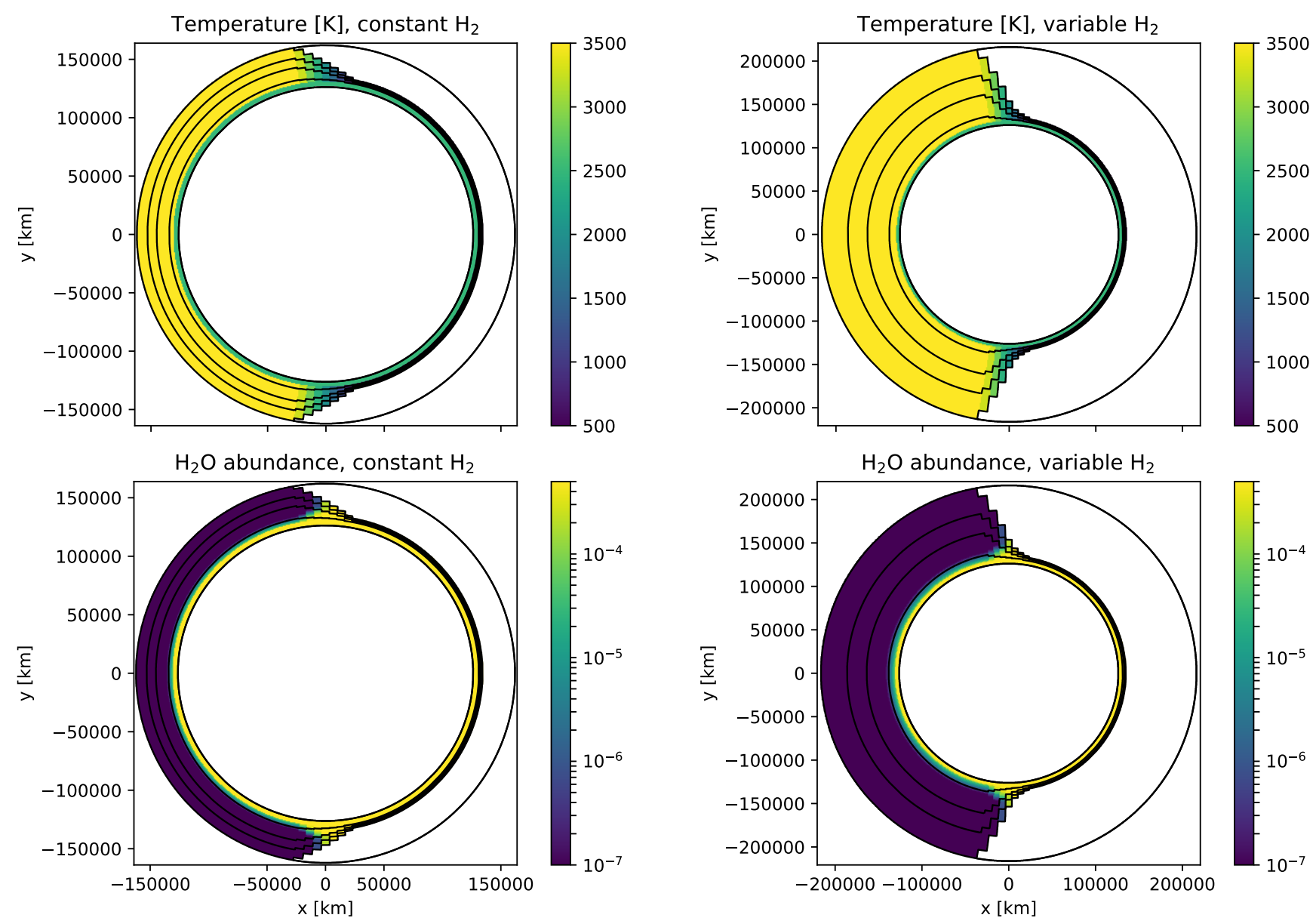

Fig. 4. Atmospheric structure of the symmetric idealized case, $\nabla^{+} T$, assuming an absence of $\mathrm{H}_{2}$ dissociation. We show the temperature map (top) and the water abundance map (bottom). The temperature gradient goes from 500 to $3500 \mathrm{~K}$. The transmission angle is $10^{\circ}$ around the terminator line and has a $2500 \mathrm{~K}$ ring at $P=0.13$ bar from the surface pressure. From the center outward, the five solid lines are, respectively, the $10^{6}, 10^{3}$, $1,10^{-2}$, and $10^{-4} \mathrm{~Pa}$ pressure levels. The color-coding for the water abundance maps goes from $5 \times 10^{-4}$ to $10^{-7}$. The radius of the planet and the atmosphere are shown to scale. The temperature and abundance maps of the $\nabla^{-} T$ case are almost the same, except the scale height of the atmosphere is smaller.

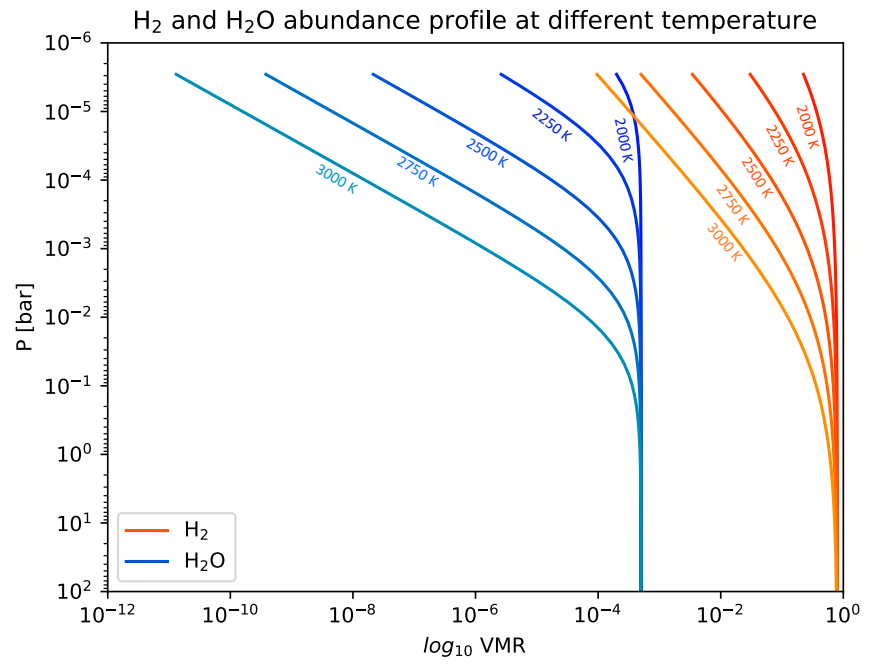

Fig. 5. Abundance profiles for $\mathrm{H}_{2} \mathrm{O}$ and $\mathrm{H}_{2}$ plotted for a range of temperature between 2000 and $3000 \mathrm{~K}$. At a given pressure, $\mathrm{H}_{2} \mathrm{O}$ and $\mathrm{H}_{2}$ dissociation depends strongly on the temperature. The higher the temperature, the lower the abundance in the atmosphere. At a fixed temperature the variation of the dissociation rate is a function of $\mathrm{P}$ and $\mathrm{P}^{2}$, respectively, for $\mathrm{H}_{2}$ and $\mathrm{H}_{2} \mathrm{O}$. spectral domain (Greene et al. 2016). It should be noted that we compute different 3D structures as input for Pytmosph3R with the parameters described in Table 1. We describe in detail these structures in Sect. 3.

\subsection{Retrieval model: TauREx}

The spectral retrievals has been computed with the TauREx retrieval code (Waldmann et al. 2015b,a). TauREx consists in a Bayesian retrieval code which uses the ExoMol line lists (Yurchenko et al. 2011, 2014; Tennyson \& Yurchenko 2012; Barton et al. 2013, 2014), HITRAN (Rothman et al. 2009; Gordon et al. 2013) and HITEMP (Gordon et al. 2010). TauREx assumes a plane-parallel exoplanetary atmosphere with 1D distribution elements and temperature-pressure profile. We assume the radius at the base of the model of Wasp-121b as the value for which the atmospheric pressure is 10 bar. We simulate the exoplanetary atmosphere assuming a pressure between $10^{6}$ and $10^{-4}$ Pascals. We consider an atmosphere dominated by hydrogen and helium, with a mean molecular weight of $2.3 \mathrm{amu}$. We also suppose the presence of $\mathrm{CO}$ and $\mathrm{H}_{2} \mathrm{O}$ as trace gases.

An important assumption is that TauREx does not take into account thermal dissociation of species and assumes that molecular abundances are constant with the atmospheric pressure 
Table 1. Parameters used in the three simulation configurations of WASP-121b.

\begin{tabular}{lccccc}
\hline \hline Input parameters & Min-max temperature $[\mathrm{K}]$ & Angle $\beta$ & $P_{\text {bot }}[\mathrm{Pa}]$ & $P_{\text {top }}[\mathrm{Pa}]$ & $x_{\mathrm{CO}}[\log (\mathrm{VMR})]$ \\
\hline Symmetric case $\nabla^{-} T$ & $1400-2800$ & $20^{\circ}$ & $10^{6}$ & $10^{-4}$ & -3.36 \\
Symmetric case $\nabla^{+} T$ & $500-3500$ & $10^{\circ}$ & $10^{6}$ & $10^{-4}$ & -3.36 \\
$\quad$ GCM case & $493-3545$ & - & $1.434 \times 10^{7}$ & $10^{-4}$ & -3.36 \\
\hline
\end{tabular}

Table 2. Mass, radius, and temperature parameters of the WASP-121 star (top) and its planet Wasp-121 b (bottom).

\begin{tabular}{|c|c|c|c|c|}
\hline Star & Mass $\left[M_{\mathrm{S}}\right]$ & Radius $\left[R_{\mathrm{S}}\right.$ & Temperature $[\mathrm{K}]$ & Bibliographical references \\
\hline Wasp-121 & 1.353 & 1.458 & 6460 & Delrez et al. (2016) \\
\hline Planet & $\operatorname{Mass}\left[M_{\mathrm{J}}\right]$ & $\operatorname{Radius}\left[R_{\mathrm{J}}\right] \quad \mathrm{T}$ & Temperature $[\mathrm{K}]$ & Bibliographical references \\
\hline asp-121 b & 1.183 & 1.8 & 2360 & zz et al. (2016), Evans et al. (2016) \\
\hline
\end{tabular}

Notes. They are used for the observable generations and for the retrievals.

(see Changeat et al. 2019 for a detailed discussion on the effect of this assumption). We set up the prior range for the molecular volume mixing ratios of $\mathrm{CO}$ and $\mathrm{H}_{2} \mathrm{O}$ between $10^{-12}$ and $10^{-1}$, which allows us to explore a wide range of solutions. Even if we never add clouds in our input simulation, we set up the prior range for clouds between $10^{-12}$ and $10^{-1}$ to allow the retrieval code to have more freedom. We also feel that this better simulates a real situation where clouds would be put in the retrieval without prior knowledge about their occurrence in the real planet. The allowed range for the planetary radius and the atmospheric temperature (assumed constant in the vertical) are, respectively, $1.3 R_{\text {Jup }}-4.0 R_{\text {Jup }}$ and $400-3600 \mathrm{~K}$, with $R_{\text {Jup }}$ the radius of Jupiter. The range for the temperature is linked to the minimum and maximum temperature in the GCM simulation of Wasp-121b, as shown in Table 1. As we simulate JWST observations, we set up the range of wavelengths between 0.6 to $10 \mu \mathrm{m}$. For the non-retrieved parameters such as masses and stellar radius, we use those describe in Table 2.

We note that the TauREx retrieval code is not designed to take into account the thermal dissociation of $\mathrm{H}_{2}$. When $\mathrm{H}_{2}$ thermal dissociation occurs, the $\mathrm{He} / \mathrm{H}_{2}$ ratio changes as a function of pressure and temperature. The mean atmospheric weight can also be lower than $2 \mathrm{amu}$. In order to be consistent across all our sets of simulations, we used the same configuration file for our spectral retrievals and we left the $\mathrm{He} / \mathrm{H}_{2}$ ratio constant. By doing so we could estimate whether an atmosphere where $\mathrm{H}_{2}$ dissociation does not occur can explain our input spectrum or not. We come back to this in Sect 4.2.3.

Again, some of these assumptions may seem too simplistic in view of the physics we envision in the real atmospheres of UHJs, but we think that it is important to understand how such simplistic assumptions - commonly used nowadays - can bias the conclusions from a retrieval analysis.

\section{Input models: from simple case to GCM simulations}

In order to understand and to characterize the biases due to the atmospheric 3D structure of the Wasp-121b atmosphere, we decided to start from a simple case, and to add further levels of complexity as the study progresses. Using this approach, we show how to distinguish the observed biases. We note that we used the same atmospheric composition in all our cases, which is $\mathrm{He}, \mathrm{H}_{2}, \mathrm{H}_{2} \mathrm{O}$, and $\mathrm{CO}$.

The validation of the Pytmosph3R model with TauREx was done by Caldas et al. (2019). So, we started by considering the atmospheric 3D structure of an idealized Wasp-121b. In Figs. 3 and 4 we show the 3D temperature maps used by Pytmosph3R to calculate the transmission spectrum. With this model we neglect effects that depend on the altitude, using isothermal pressure-temperature profiles in each cell, and we focus on the compositional effects. Finally, we simulated a 3D atmospheric structure of Wasp-121b using a GCM that takes into account both vertical and horizontal effects.

\subsection{Idealized 3D atmosphere with day to night gradient}

\subsubsection{Effects of the day to night temperature gradient}

First, we create an idealized case of Wasp-121b atmospheric structure that is symmetric around the planet-observer line of sight. The main parameters to simulate this simple structure are $T_{\mathrm{d}}, T_{\mathrm{n}}$, and $\beta$, which are respectively the day-side and night-side temperature, and the opening angle. $T_{\mathrm{d}}$ and $T_{\mathrm{n}}$ are correlated respectively with the substellar and the antistellar point. The opening angle $\beta$ describes how the transitions between the day and the night side are computed: the smaller, the sharper. We constructed our model by going symmetrically from the dayside to the night-side temperature considering a linear transition between the two. To avoid effects due to vertical temperature gradients as much as possible, every column of our model follows an isothermal profile above a given pressure level $\left(P_{\text {iso }}=0.13 \mathrm{bar}\right)$. In this part of the atmosphere, however, the temperature varies with respect to the local solar elevation angle, $\alpha_{*}$. In the deeper parts of the model, in agreement with results from a GCM for Wasp-121b (see Sect. 2.1), the temperature is uniform and set to $2500 \mathrm{~K}$. In summary, for a given pressure and local solar elevation angle, the temperature is given by

$$
\left\{\begin{aligned}
P>P_{\text {iso }} & T=T_{\mathrm{d}} \\
P<P_{\text {iso }} & \left\{\begin{aligned}
2 \alpha_{*}>\beta & T=T_{\mathrm{d}} \\
\beta>2 \alpha_{*}>-\beta & T=T_{\mathrm{n}}+\left(T_{\mathrm{d}}-T_{\mathrm{n}}\right) \frac{\alpha_{*}+\frac{\beta}{2}}{\beta} \\
2 \alpha_{*}<-\beta & T=T_{\mathrm{n}}
\end{aligned}\right.
\end{aligned}\right.
$$


For Wasp-121b we considered two end-member cases:

1 . The high gradient model $\left(\nabla^{+} T\right)$, which goes from 500 to $3500 \mathrm{~K}$ using $\beta=10^{\circ}$. This case is supposed to be representative of the day-night gradient present in the GCM without the heterogeneities in the vertical direction and along the limb.

2 . The low gradient model $\left(\nabla^{-} T\right)$, which goes from 1400 to $2800 \mathrm{~K}$ using $\beta=20^{\circ}$. This case is more representative of GCM simulations accounting for the thermal effect of $\mathrm{H}_{2}$ recombination. We anticipate this case to be conservative and to provide a lower bound for the real bias caused by day-night temperature differences.

Figures 3 and 4 show the temperature structure of the atmosphere of the cases studied. These maps are plotted to scale. We note that we consider a symmetric atmospheric structure, so the equatorial cut and the polar cut are exactly the same. As we can see, the day sides of the atmospheres are strongly inflated in comparison to the night sides, which creates the horizontal biases that we want to characterize.

The $\nabla^{+} T$ and $\nabla^{-} T$ cases have been chosen to encompass the real day-night gradient present in Wasp-121b (see below), while removing the effects due to differences along the limb (e.g., east vs. west limb or equator vs. poles). This should allow us to identify the specific effect of these differences by comparing the retrieval results with our idealized cases and with the actual GCM outputs.

The $\nabla^{+} T$ case is close to the GCM case. Bell \& Cowan (2018) and Tan \& Komacek (2019) showed that the energy redistribution effect of hydrogen recombination cools the day side, heats the night side, and reduces the gradient across the limb. The $\nabla^{-} T$ case thus uses a reduced gradient to account for this effect and present a very conservative estimate of the 3D effect.

\subsubsection{Composition effects due to the dissociation of some molecules}

In UHJs, molecular abundances are not the same everywhere, since the day-side temperature is high enough to allow thermal dissociation of many species (Lodders \& Fegley 2002; Visscher et al. 2006, 2010; Marley et al. 2017). In this work, we take into account the dissociation of two molecular species, first only $\mathrm{H}_{2} \mathrm{O}$ and later also $\mathrm{H}_{2}$, using the equations provided by Parmentier et al. (2018), as shown in Fig. 5. At a given temperature, the lower the pressure, the stronger the dissociation and at a given pressure, the higher the temperature, the higher the dissociation. In this study, we assume that dissociated $\mathrm{H}_{2}$ predominantly forms atomic hydrogen. We know that hydrogen anions can absorb part of the electromagnetic radiation between 1 and $10 \mu \mathrm{m}$ (Lenzuni et al. 1991; Parmentier et al. 2018). However, we did not include in our computations the absorption contribution from ionized hydrogen. The main reason is that we want to be consistent with TauREx, which does not include this opacity source. Furthermore, the dissociation of $\mathrm{CO}$ cannot occur at the temperature regimes in our simulations $(500-3500 \mathrm{~K})$. In order to dissociate, $\mathrm{CO}$ requires higher temperatures, due to its stronger triple bond (Lodders \& Fegley 2002). For this reason, in all the simulations we assumed a constant $\mathrm{CO}$ abundance everywhere in the atmosphere. In the first set of simulations we considered the $\nabla^{+} T$ and $\nabla^{-} T$ models considering water dissociation alone $\left(\mathrm{H}_{2}\right.$ is held constant). As shown in the abundance map of $\mathrm{H}_{2} \mathrm{O}$ in Figs. 3 and 4, there is no more water on the day side of the planet at pressure lower than $P_{\text {iso }}$. The dissociation stops around the terminator, and on the night side the water distribution comes back at a constant value.
In the second set of simulations we took into account both $\mathrm{H}_{2}$ and $\mathrm{H}_{2} \mathrm{O}$ dissociation. As $\mathrm{H}_{2}$ does not have significant absorption bands in the $0.6-10 \mu \mathrm{m}$ wavelength range, the main effects of its dissociation is to decrease the molecular weight, causing an increase in the atmospheric scale height. It also affects the thermochemical properties of the atmosphere because atomic hydrogen recombines on the night side. This redistribution of energy will heat the night side and cool the day side (Bell \& Cowan 2018; Tan \& Komacek 2019). Another important effect is that without $\mathrm{H}_{2}$ in the atmosphere, collision induced effects from the $\mathrm{H}_{2}-\mathrm{H}_{2}$ and $\mathrm{He}-\mathrm{H}_{2}$ collisions are both less significant. As shown in Figs. 3 and $4, \mathrm{H}_{2}$ dissociation increases the atmospheric scale height of the day side by a factor of 1.5 and 2 in the $\nabla^{-} T$ and $\nabla^{+} T$ simulations, respectively. We note that the temperature is not high enough to induce $\mathrm{H}_{2}$ dissociation on the night side. This characteristic creates an even stronger difference between the night side and the day side when $\mathrm{H}_{2}$ dissociates. Consequently, $\mathrm{CO}$ is the dominant radiatively active species on the day side because it does not dissociate at these temperatures.

\section{2. $3 D$ models of the Wasp-121b atmosphere using SPARC/MITgcm global circulation model}

In the last configuration we add both the vertical and azimuthal (along the limb) effects. We model Wasp-121b using the GCM (SPARC/MITgcm; Parmentier et al. 2018) described in Sect 2.1. The chemistry is kept unchanged. As in Sect. 3.1, we studied four different cases considering all the possible combinations where water and $\mathrm{H}_{2}$ dissociate. Figures 1 and 2 show the temperature and water abundance maps for the GCM cases with and without $\mathrm{H}_{2}$ dissociation. We see that the thermal structure of the atmosphere is more complex than in the symmetric case, even if the salient features remain the same. The most important modification concerns the terminator, which is now asymmetric because of the winds created by the strong temperature dichotomy between the day and night side (Showman et al. 2015). These equatorial jets warm the east side of the atmosphere and cool the west side around the equator, which implies less dissociation of water on the west because of the cooler temperature. Nevertheless, the abundance maps show that there is still water dissociated at the terminator, which could allow the light to probe deeper into the atmosphere on the night side of the planet. We also note that when $\mathrm{H}_{2}$ dissociation is taken into account, the global behavior of the 3D structure remains the same, but since the day side of the atmosphere has a greater scale height, it is more inflated.

An important caveat here is that while we model $\mathrm{H}_{2}$ dissociation to compute the transit spectrum, the dissociation is not taken into account in the GCM itself. This is expected to reduce the day-night contrast, and thus the wind fields (Bell \& Cowan 2018; Tan \& Komacek 2019). So this case is not completely self-consistent.

\section{Results}

In this section we describe the results of the transmission spectra generated by Pytmosph3R and the spectral retrieval using our three sets of simulations, each one being performed on our three temperature structures $\left(\nabla^{-} T, \nabla^{+} T\right.$, and GCM):

1. $\mathrm{H}_{2}$ and $\mathrm{H}_{2} \mathrm{O}$ constant in the whole atmosphere;

2. thermal dissociation of $\mathrm{H}_{2} \mathrm{O}$ with $\mathrm{H}_{2}$ held constant;

3. thermal dissociation of both $\mathrm{H}_{2} \mathrm{O}$ and $\mathrm{H}_{2}$. 


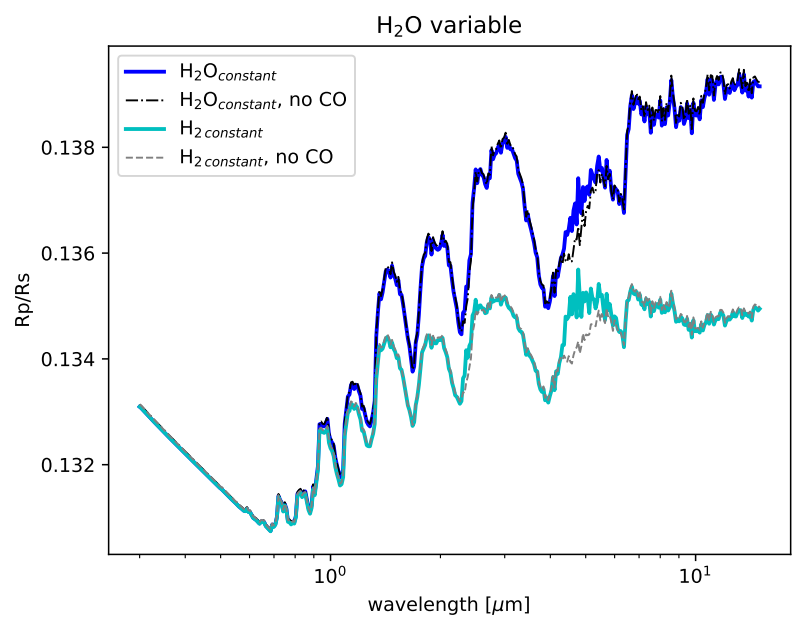

(a)

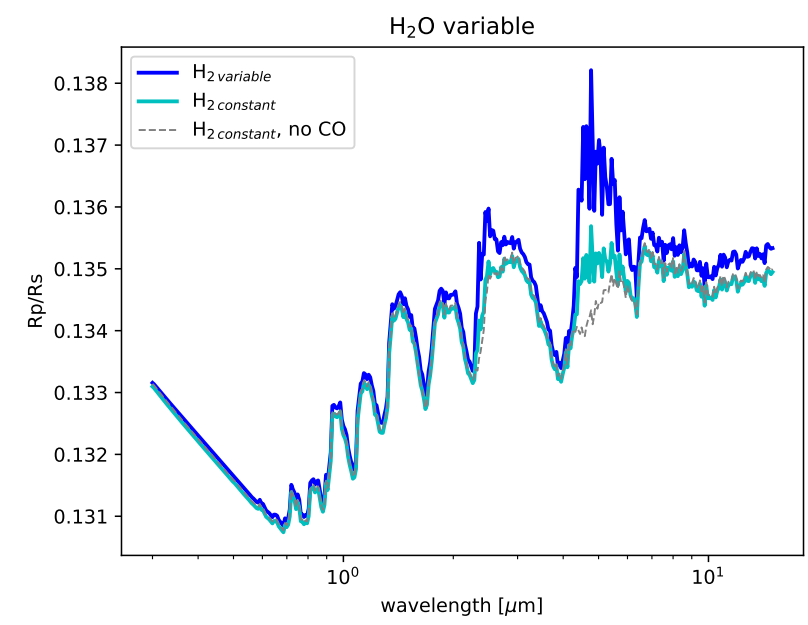

(b)

Fig. 6. Left: transmission spectra of Wasp-121b at a resolution of $R=100$ for GCM simulations assuming a constant $\mathrm{H}_{2}$ abundance in the whole atmosphere. When water dissociation is taken into account (light blue line), the water features become shallower compared to when no water dissociation is assumed (blue line). Right: transmission spectra of Wasp-121b at a resolution of $R=100$ for GCM simulations taking into account $\mathrm{H}_{2} \mathrm{O}$ dissociation in the atmosphere. When $\mathrm{H}_{2}$ dissociation is considered (blue line), the CO features appear more clearly compared to when we neglect $\mathrm{H}_{2}$ dissociation (light blue line). The black and gray curve correspond to the transmission spectra for an atmosphere without $\mathrm{CO}$ for the water constant and the dissociated case, respectively, to highlight the features of $\mathrm{CO}$ in the other curves.

\section{1. $3 D$ transmission spectrum of ultra-hot Jupiters}

The transmission spectra generated by Pytmosph3R are shown in Fig. 6. Here we tested two cases with $\mathrm{H}_{2}$ constant considering or not water dissociation in the atmosphere (Fig. 6a), and a third one with both $\mathrm{H}_{2} \mathrm{O}$ and $\mathrm{H}_{2}$ dissociation (Fig. 6b). When $\mathrm{H}_{2} \mathrm{O}$ is constant, the water features are more evident and the $\mathrm{CO}$ features are barely visible as it is highlighted comparing the transmission spectra with and without $\mathrm{CO}$ in the atmosphere (respectively the blue and the dotted black lines). Since there is $\mathrm{CO}$ and $\mathrm{H}_{2} \mathrm{O}$ everywhere, then the transmitted light probes the same region for both molecules, which are at a high altitude on the day side of the atmosphere. In this case then the spectral features are similar to those of the $1 \mathrm{D}$ case.

When $\mathrm{H}_{2} \mathrm{O}$ dissociates (light blue curve in Fig. 6a), the water features in the spectrum become smaller because it almost disappears from the day side of the atmosphere. Then the light from the star can probe much deeper regions of the atmosphere in the wavelength range where the $\mathrm{CO}$ does not absorb and reaches part of the night side. The $\mathrm{CO}$ is not dissociated on the day side, where the scale height is large due to the high temperature, and appears more evidently. So, as the light goes first through the day side and then through the night side, the transmission spectrum carries information of both sides of the atmosphere depending on the wavelength being looked at. The behavior of the spectrum can also be understood looking at Figs. 1- 4 where the maps of the atmosphere show the lack of water on the day side except for a deep part of the atmosphere $(\simeq 10$ mbar). Since CO does not dissociate, it absorbs mainly on the day side of the atmosphere where the high temperature leads to larger absorption features.

When both $\mathrm{H}_{2} \mathrm{O}$ and $\mathrm{H}_{2}$ dissociate, the water features are similar in both the presence and absence of $\mathrm{H}_{2}$ dissociation, as shown in Figs. 5 and 6b. The main effect of the dissociation of $\mathrm{H}_{2}$ in the atmosphere is the increase in scale height. As the dissociation of $\mathrm{H}_{2}$ mainly occurs on the day side of the atmosphere, as does the dissociation of $\mathrm{H}_{2} \mathrm{O}$ (see Figs. 1 and 2), the region probed is the same in both cases. However, the $\mathrm{CO}$ features appears even more clearly. As the $\mathrm{CO}$ abundance remains constant everywhere in the atmosphere, its features are more significant due to the enlarger of the scale height caused by the dissociation of $\mathrm{H}_{2}$ on the day side.

It should be noted that Fig. 6 represents the transmission spectra for the case with the GCM input, but all the behaviors explained above remain similar to the symmetric $\nabla^{+} T$ and $\nabla^{-} T$ cases.

\subsection{Retrieval results}

\subsection{1. $\mathrm{H}_{2}$ and $\mathrm{H}_{2} \mathrm{O}$ constant in the atmosphere}

The posterior distributions of $\nabla^{+} T$ and GCM simulations are shown in Fig. 7. The retrieval code TauREx converges, in every simulation, to a non-degenerate solution. Figure 8 summarizes all the solutions found by TauREx for each simulation. As shown in the red part of the various panels of this figure, TauREx finds non-degenerate solution within $2 \sigma$ from the input $\mathrm{H}_{2} \mathrm{O}$ and $\mathrm{CO}$ abundance for the $\nabla^{+} T, \nabla^{-} T$, and GCM simulations. These chemically homogeneous simulations are similar to what was done in Caldas et al. (2019), which is why we expected to find consistent results with their work. To better interpret those results, we plotted the $[\mathrm{CO}] /\left[\mathrm{H}_{2} \mathrm{O}\right]$ ratio (Fig. 8). This ratio allows us to understand if the dissociation of water affects the spectrum. The $[\mathrm{CO}] /\left[\mathrm{H}_{2} \mathrm{O}\right]$ ratio is constant when water does not dissociate, which is found here by TauREx retrieval. The temperatures retrieved suggest that the light is probing the day side of the atmosphere. Indeed, as shown in Figs. 4 and 1, the atmosphere presents an inflated day side. The temperature retrieved in the $\nabla^{+} T$ case is higher than that retrieved in the $\nabla^{-} T$ case, which is expected since the temperatures on the day side are higher in the $\nabla^{+} T$ case. Using as input the spectrum computed with GCM, TauREx finds a slightly lower temperature than the value found in the two symmetric simulations. Considering that the GCM simulations were made assuming a non-isothermal temperature-pressure profile, the temperature structure of GCM is indeed more complex than that in the other two simulations. These results are all consistent with Caldas et al. (2019). We note that the calculated $\chi^{2}$ is much higher for the GCM case compared to the symmetric cases $\nabla^{+} T$ and $\nabla^{-} T$. 
W. Pluriel et al.: Strong biases in retrieved atmospheric composition caused by strong day-night chemical heterogeneities
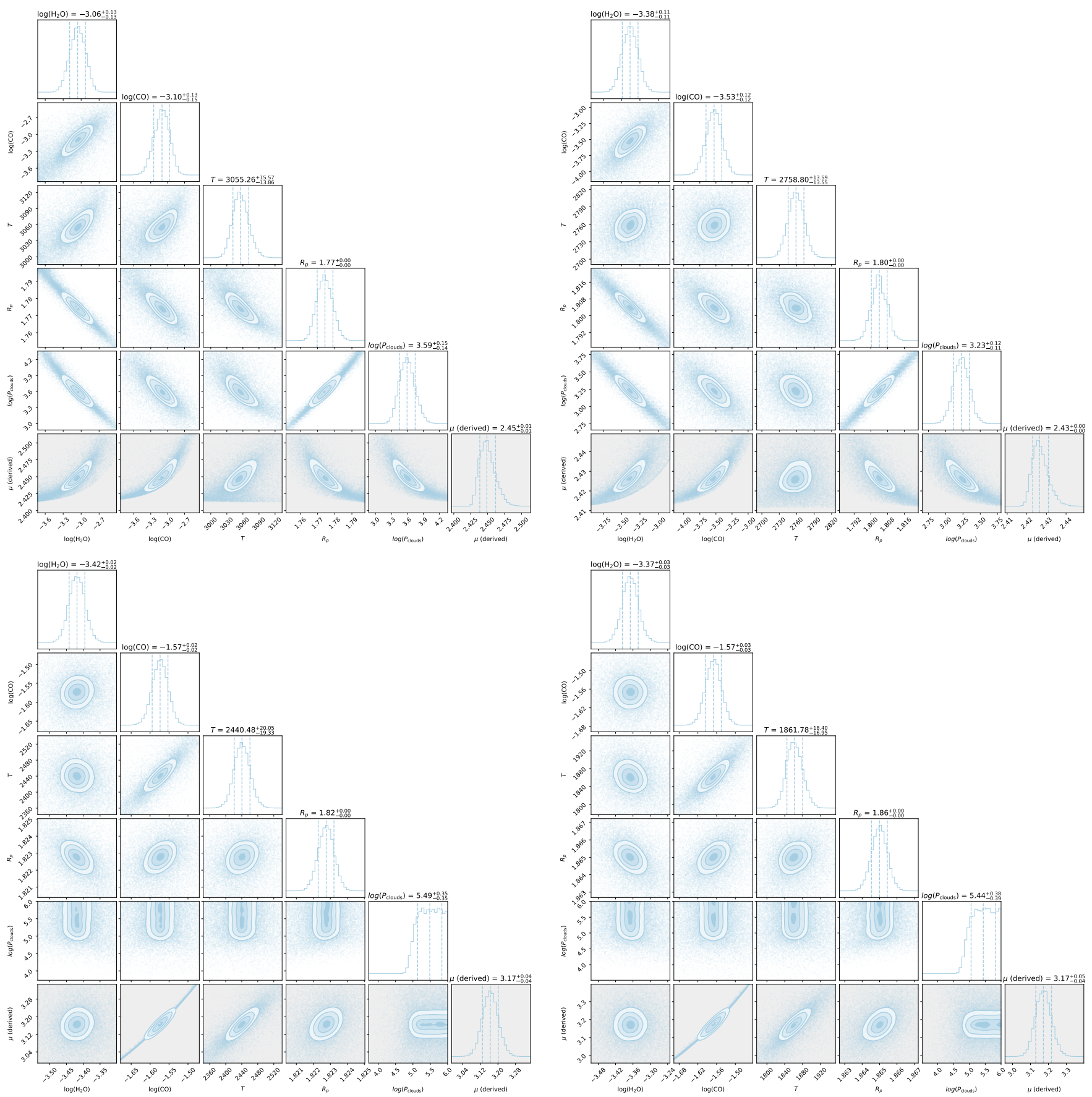

Fig. 7. Nest posteriors retrieval from TauREx assuming a constant $\mathrm{H}_{2}$ abundance in the atmosphere. Shown are the $\nabla^{+} T$ (left) and GCM cases (right), which assume, respectively, no water dissociation (top) and water dissociation (bottom). We retrieved five free parameters, which are $\mathrm{H}_{2} \mathrm{O}$, $\mathrm{CO}$ abundances in $\log 10(\mathrm{VMR})$, clouds pressure in bar, the temperature in Kelvin, and the planetary radius in Jupiter's radius. The molecular weight is derived from these parameters.

\subsubsection{Thermal dissociation of $\mathrm{H}_{2} \mathrm{O}$ with $\mathrm{H}_{2}$ constant}

When water dissociation is taken into account, TauREx returns significantly different solutions compared to the homogeneous simulations. The results are shown in the yellow parts of Fig. 8 .

First, all the retrieved temperatures are lower than the values found in the constant composition simulations. Because the main constraint that allows TauREx to retrieve the temperature is the amplitude of the spectral bands (the higher the temperature, the higher the amplitude), the temperatures retrieved are lower because the water features are coming from a colder region of the atmosphere. In the GCM case the temperature retrieved is lower than the temperatures of the limb, which suggest that we are probing the night side of the atmosphere.

Since CO does not dissociate, it absorbs mainly on the day side of the atmosphere where the high temperature leads to larger absorption features, as explained in Sect 4.1. Looking at the yellow part of Fig. 8, there are non-degenerate solutions for TauREx to fit the $\mathrm{CO}$ features, considering that it also finds a low temperature because of the presence of water on the cold night side of the planet. This solution suggests a high $\mathrm{CO}$ abundance in the atmosphere and converges around the correct water abundance within $3 \sigma$. This water abundance corresponds to the 

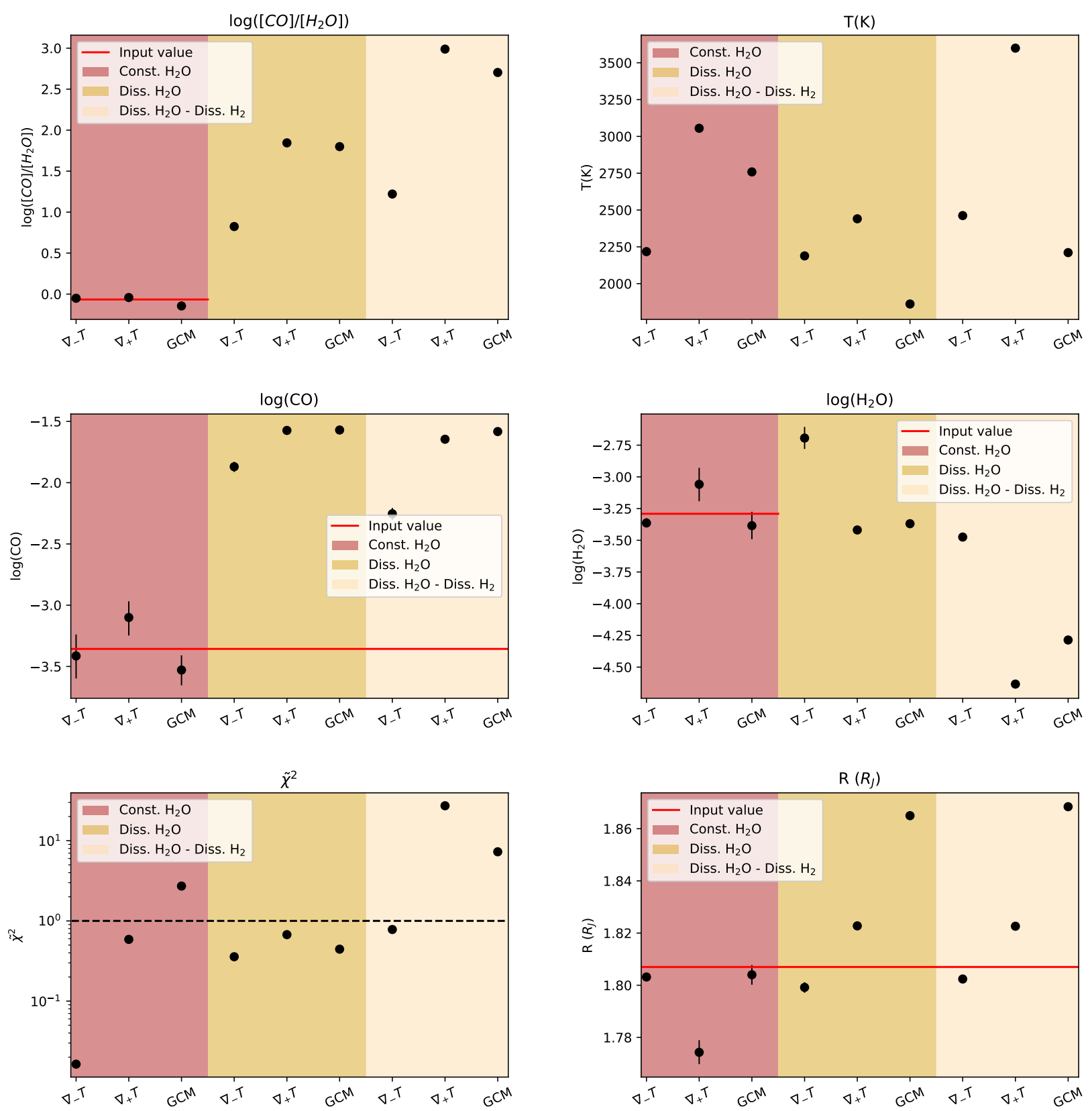

Fig. 8. Summary of all the retrieval results for the $\nabla^{-} T, \nabla^{+} T$, and GCM simulations considering every case. We show the ratio $[\mathrm{CO}] /\left[\mathrm{H}_{2} \mathrm{O}\right](t o p$ left), temperature (top right), log abundances of $\mathrm{CO}$ (middle left) and $\mathrm{H}_{2} \mathrm{O}$ (middle right), $\tilde{\chi}^{2}$ (bottom left), and planetary radius (bottom right). These retrievals have been calculated with a shot noise assuming a floor noise of 30ppms through the whole spectral domain. The red line represents the input value from our simulations and the black dotted line shows where $\tilde{\chi}^{2}=1$.

abundance on the night side of the atmosphere where water is not dissociated (see Figs. 1, 3, and 4).

As a consequence, the $[\mathrm{CO}] /\left[\mathrm{H}_{2} \mathrm{O}\right]$ is around 100 times higher than expected. We note that for the conservative case $\nabla^{-} T$ the $[\mathrm{CO}] /\left[\mathrm{H}_{2} \mathrm{O}\right]$ is less biased, around 10 times higher than the solar abundance, due to the smaller day to night contrast and the smoother transition between them. To quantify the reliability of the solutions given by TauREx, we plotted the absorption contribution plots of the solutions for the GCM case and the differences in ppm between the generated and the retrieved spectrum in Fig. 9. We note here in which wavelength bands the fit is good or not. Moreover, the calculated $\tilde{\chi}^{2}$, which is below a value of 1 for each simulation, demonstrates a high significance level of the solution given by TauREx (see Fig. 8).

\subsubsection{Thermal dissociation of $\mathrm{H}_{2}$ and $\mathrm{H}_{2} \mathrm{O}$}

In Fig. 8 we give the $\tilde{\chi}^{2}$ values for each simulation. We see that TauREx finds a non-degenerate solution for every simulation, although they are not all statistically significant (e.g., $\tilde{\chi}^{2} \gg 1$ in the $\nabla^{+} T$ and GCM cases). In the previous cases where we have a constant composition in the entire atmosphere and in the case where only water dissociates on the day side, TauREx finds a consistent solution even though it does not necessarily converge toward the correct input parameters. On the contrary, when $\mathrm{H}_{2}$ dissociation is taken into account, TauREx cannot find a suitable solution to explain the atmospheric spectrum. As shown in the lower left panel of Fig. 8, the best reduced $\chi^{2}$ for the $\nabla^{+} T$ and GCM models with $\mathrm{H}_{2}$ dissociation are respectively around 30 and 8 . The $\chi^{2}$ test can give us a possible signal when $\mathrm{H}_{2}$ 

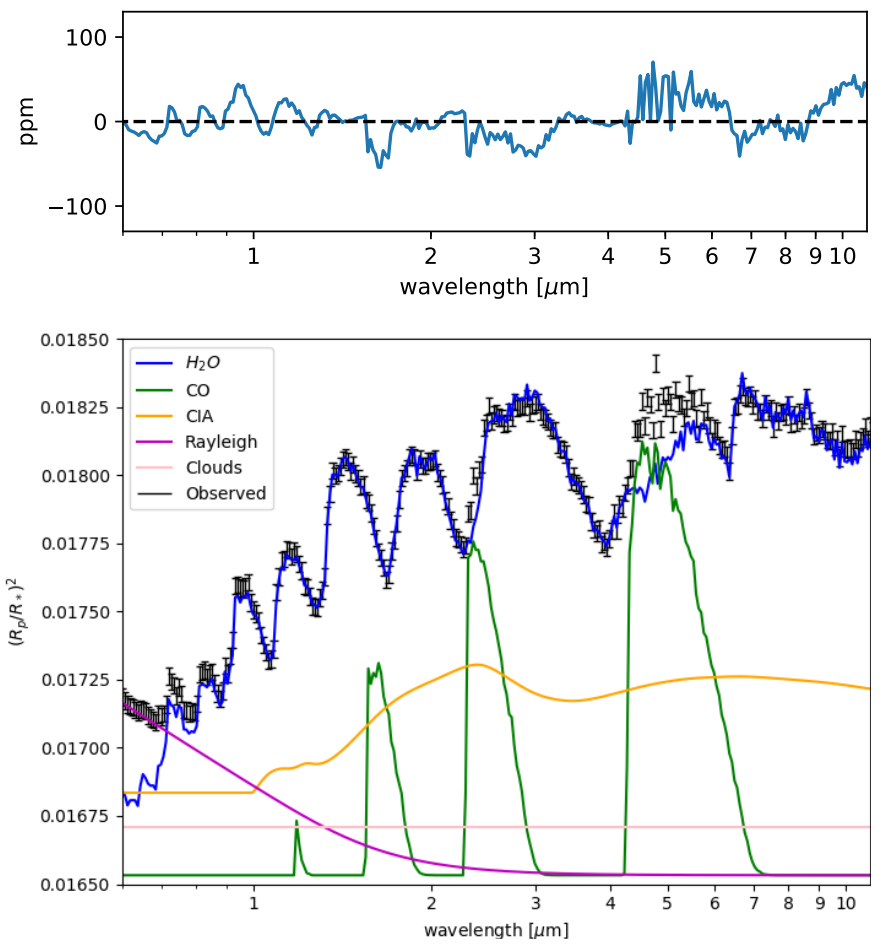

Fig. 9. Particular solutions found by TauREx in the GCM case assuming water dissociation and $\mathrm{H}_{2}$ constant. Bottom: contribution plots of each component, i.e., $\mathrm{H}_{2} \mathrm{O}, \mathrm{CO}$, and collision induced absorption (CIA) for the solution found by TauREx. Top: residuals (in ppm) between the input spectrum and the best fit spectrum.

dissociation occurs in the planet we are considering. However, TauREx manages to find a statistically significant solution in the $\nabla^{-} T$ case; however, keeping in mind that this simulation is a conservative choice, we think that the truth remains between the $\nabla^{-} T$ and $\nabla^{+} T$ simulations.

We note that the TauREx retrieval code is not designed to take into account the thermal dissociation of $\mathrm{H}_{2}$. When $\mathrm{H}_{2}$ dissociation occurs, the $\mathrm{He} / \mathrm{H}_{2}$ ratio changes as a function of pressure and temperature. The mean atmospheric weight can also be lower than 2amu in the atmospheric regions where $\mathrm{H}_{2}$ dissociates. In order to be consistent with all the sets of simulations, we used the same configuration file for our spectral retrievals and we left the $\mathrm{He} / \mathrm{H}_{2}$ ratio constant. By doing so we were able to estimate whether an atmosphere where $\mathrm{H}_{2}$ dissociation does not occur can explain our input spectrum or not.

The $\mathrm{H}_{2}$ dissociation plays a major role on the spectra and the retrieval results. All the results are shown in the light yellow part of Fig. 8. In each simulation, the $[\mathrm{CO}] /\left[\mathrm{H}_{2} \mathrm{O}\right]$ ratio retrieved is higher than the value calculated when $\mathrm{H}_{2}$ is constant. As $\mathrm{CO}$ abundance remains constant everywhere, its features are then more significant, as explained in Sect. 4.1, and TauREx finds a best fit model with a low water abundance and a high $\mathrm{CO}$ abundance. As a consequence, the $[\mathrm{CO}] /\left[\mathrm{H}_{2} \mathrm{O}\right]$ ratio increases.

As shown in the light yellow part in Fig. 8, when we consider $\mathrm{H}_{2}$ dissociation in the GCM case TauREx finds non-degenerate solution. The temperature retrieved is a bit higher than the temperature we find when $\mathrm{H}_{2}$ does not dissociate. As shown in Fig. 6, $\mathrm{H}_{2}$ dissociation does not alter the amplitude of the water features dramatically because the water absorption comes from the cold night side of the planet where molecular $\mathrm{H}_{2}$ dominates. Thus, the temperature retrieved still corresponds to the temperature of the night side.
For the other retrieved parameters, we are consistent with the solution found when $\mathrm{H}_{2}$ is constant. We remember that the dissociation of $\mathrm{H}_{2}$ affects the transmission spectrum in two main ways. First, it increases the scale height of the day side of the atmosphere implying stronger features for species that remain on the day side (such as $\mathrm{CO}$ ). We only consider this effect in the $\nabla^{+} T$ simulation. Second, it also significantly affects the thermochemical properties of the atmosphere due to the recombination of $\mathrm{H}_{2}$ on the night side, which redistributes the energy, heating the night side and cooling the day side (Bell \& Cowan 2018; Tan \& Komacek 2019). We took into account both of them in the $\nabla^{-} T$ simulation. $\mathrm{H}_{2}$ dissociation also results in a decrease in $\mathrm{H}_{2}-\mathrm{H}_{2}$ and $\mathrm{He}-\mathrm{H}_{2}$ collisions, hence less intense continuum absorptions. However, in the $\nabla^{+} T$ simulations TauREx does not manage to converge to a reasonable solution. TauREx cannot explain the line distortion using a 1D atmospheric model, and tends to increase the temperature far above the equilibrium temperature of the planet, reaching the upper prior range temperature of $3600 \mathrm{~K}$ (see Fig. 8).

When both $\mathrm{H}_{2}$ and $\mathrm{H}_{2} \mathrm{O}$ dissociate, it is not possible to find any $1 \mathrm{D}$ atmospheric model that matches the input spectrum in any of the cases under study in this paper.

\section{Discussions}

Our results show that a $1 \mathrm{D}$ model is always able to find a statistically consistent solution, except for the case in which $\mathrm{H}_{2}$ dissociation occurs, although it does not always converge toward the correct input solution.

Caldas et al. (2019) showed that day-night temperature contrasts lead to a bias in retrieved temperatures (toward that of the day side) even when the chemical composition is uniform. In this work we extend this idea by showing that when we take into account $\mathrm{H}_{2} \mathrm{O}$ dissociation we have both composition and temperature biases.

Although a retrieval code can use a 1D atmospheric model to detect the presence of a particular chemical species from an atmosphere with a complex 3D structure, measuring its abundance is more challenging. When temperature and chemical composition vary across the limb, the 1D retrieval cannot find the correct molecular abundances: the best fit parameters can be several orders of magnitude different from the correct input values, as shown in Fig. 8.

Elemental ratios (e.g., C/O, $\mathrm{C} / \mathrm{N}$ ) are key parameters determining the chemical processes taking place in planetary atmospheres (Line et al. 2013; Oreshenko et al. 2017). At high temperature, Madhusudhan (2012) and Espinoza et al. (2017) show that $\mathrm{C} / \mathrm{O}$ plays a major role in the atmospheric chemistry and non-solar values could explain observations for six hot Jupiters (XO-1b, CoRoT-2b, WASP-14b, WASP-19b, WASP$33 \mathrm{~b}$, and WASP-12b). However, these elemental ratios cannot be directly measured, and we have to rely on measuring the abundance values of all molecules carrying the considered elements. In the case of the hot hydrogen-dominated atmospheres considered here, $\mathrm{H}_{2} \mathrm{O}$ and $\mathrm{CO}$ are the main carriers of carbon and oxygen so that $[\mathrm{CO}] /\left[\mathrm{H}_{2} \mathrm{O}\right]$ is commonly expected to provide reasonable constraints on $\mathrm{C} / \mathrm{O}$. Our study shows that using $[\mathrm{CO}] /\left[\mathrm{H}_{2} \mathrm{O}\right]$ to constrain $\mathrm{C} / \mathrm{O}$ when dissociation is present is highly hazardous and that finding $[\mathrm{CO}] /\left[\mathrm{H}_{2} \mathrm{O}\right] \gg 1$ should be seen as a sign of chemical heterogeneities in the atmosphere. We note that the same diagnostic could be applied to other species such as $\mathrm{TiO}$ or $\mathrm{VO}$ by calculating the retrieved ratios $[\mathrm{CO}] /[\mathrm{TiO}]$ or $[\mathrm{CO}] /[\mathrm{VO}]$. 
Our retrieval results are consistent for both the GCM model and our more idealized models of WASP-121b where temperature is constant in the vertical and symmetric around the substellar point, which reveals (for UHJs at least) that the effect of thermal and compositional heterogeneities across the limb dominate over the vertical ones, and over those along the limb. Fixing an atmospheric configuration (i.e., in presence or not of $\mathrm{H}_{2}$ and/or $\mathrm{H}_{2} \mathrm{O}$ dissociation; see Fig. 8) the retrieved values of $[\mathrm{CO}] /\left[\mathrm{H}_{2} \mathrm{O}\right]$ for the $\nabla^{+} T, \nabla^{-} T$, and GCM simulations are almost the same. This result shows that the idealized cases capture the most salient features of the GCM simulation. The most important point is that the retrieved value of $[\mathrm{CO}] /\left[\mathrm{H}_{2} \mathrm{O}\right]$ reaches its minimum when molecular dissociation does not occur; instead it increases by a factor of 10-100 when $\mathrm{H}_{2} \mathrm{O}$ dissociates and by a factor of 25-1000 when $\mathrm{H}_{2}$ dissociates as well, irrespective of the details of the vertical structure of equator-to-pole thermal contrasts. This large scale in $[\mathrm{CO}] /\left[\mathrm{H}_{2} \mathrm{O}\right]$ retrieved is due to our idealized $\nabla^{+} T$ and $\nabla^{-} T$ simulations and represents extreme cases, thus they encompass the biases.

A general characteristic of our results is that, in all the cases under our study, we cannot converge around the ground truth parameters. When we study a transmission spectrum extracted from a 3D atmosphere, even a high signal-to-noise spectrum does not allow us to know the real chemical distribution of elements with a retrieval code that uses a 1D atmospheric approximation.

A second aspect is that with a $\tilde{\chi}^{2}$-test it is possible to understand whether there is some more complex physical phenomenon in the atmosphere under study. In our case we see that a value of $\tilde{\chi}^{2}>1$ was due to the presence of $\mathrm{H}_{2}$ dissociation in our atmosphere. From this point of view, a $\tilde{\chi}^{2}$-test can reveal whether there is an important physical phenomenon that we are not considering.

It is important to note that a limitation of our study concerns the chemical components of the atmosphere. We only consider two absorber species, $\mathrm{CO}$ and $\mathrm{H}_{2} \mathrm{O}$, but we know that other species have been detected in Wasp-121b, such as TiO or VO. Furthermore, we also know that our model gives temperatures that are high enough to dissociate those species (Parmentier et al. 2018) which would add more complexity in the retrieval analysis.

\section{Are there hints of 3D structures in real data?}

In Fig. 10 we compare the spectrum of Wasp-121b as observed with HST/WFC3 (Evans et al. 2016) with two sets of synthetic transmission spectra: (i) the models developed by Parmentier et al. (2018) that extract the columns at the terminator of their GCM simulation to compute the transmission spectrum, and (ii) our models based on the same GCM simulation but with our more complete $3 \mathrm{D}$ radiative transfer framework. In addition to the radiatively active species discussed above, we account here for absoptions by $\mathrm{TiO}, \mathrm{VO}, \mathrm{Na}$, and $\mathrm{H}^{-}$using the analytical fit for the thermal dissociation of the species in Parmentier et al. (2018). Compared to Parmentier et al. (2018), only FeH is missing, but it is believed to be less important in this part of the spectrum since we assume a solar composition.

In order to fit HST/WFC3 data, Parmentier et al. (2018) suggests adding $\mathrm{CaTiO}_{3}$ clouds in the atmosphere to increase the absorption at $1.25 \mu \mathrm{m}$ and to fill the water window region. From our analysis, we see that considering a cloud-free 3D structure can equivalently explain water amplitude in the WFC3 bandpass (thick blue line) even if our spectrum does not fit the data very well either. Three-dimensional effects can indeed deform the shape of the spectrum; however, the reason is quite different.

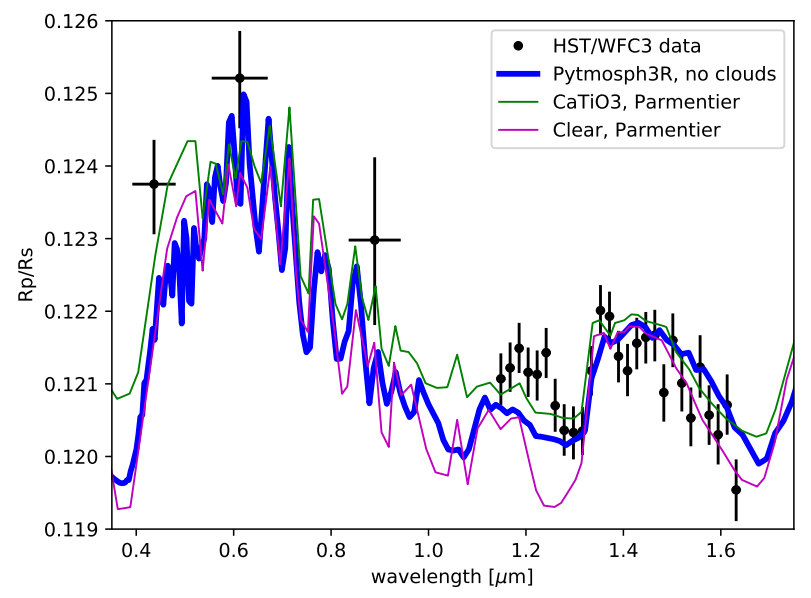

Fig. 10. Transmission spectra of Wasp- $121 \mathrm{~b}$ at resolution $R=100$ for an atmosphere composed of $\mathrm{He}, \mathrm{H}_{2}, \mathrm{H}_{2} \mathrm{O}, \mathrm{CO}, \mathrm{TiO}, \mathrm{VO}, \mathrm{Na}$, and $\mathrm{H}^{-}$ using the analytical fit for the thermal dissociation of the species in Parmentier et al. (2018; blue). Shown is a comparison of the models with HST/WFC3 data taken from Evans et al. (2016; black dot) and with two models from Parmentier et al. (2018), the first without clouds (magenta) and the second with $\mathrm{CaTiO} 3$ clouds (green).

Instead of fitting the $1.25 \mu \mathrm{m}$ window region, here the agreement is reached by reducing the strength of the 1.15 and $1.4 \mu \mathrm{m}$ water bands thanks to water removal on the day side of the atmosphere. The fact that both models can have the same observed transit depth at these two wavelengths in Fig. 10 is just due to the fact that the radius at the base of the model remains a free parameter allowing us to shift the whole spectra vertically. We note that, except for this parameter, there is no fitting of the abundances or thermal structure involved in our approach. We show that because of the water dissociation, 3D effects were non-negligible for atmospheres as hot as Wasp-121b, even if clouds remain a reasonable assumption to fit the data. This findings suggests that 3D heterogeneities in the transmission spectra and in the clouds can be combined to explain the reduction of the water feature. However, this depends on the altitude at which the clouds remain and where they are in the atmosphere; even if there are clouds, the light rays could not probe them due to the $3 \mathrm{D}$ structure of the atmosphere.

\section{Conclusion}

The 3D structure of the planetary atmospheres plays a major role in shaping of the transmission spectra, especially for ultrahot Jupiters. The tidally locked planets have an inflated day side due to both the high temperature and the dissociation of species which increase the scale height of the day-side atmosphere. In these atmospheres, the transmitted spectrum is affected by an extended region around the terminator line, which includes part of both the day side and the night side of the atmosphere.

The stellar light probes an atmospheric region which extends significantly toward the limb, depending on the chemical composition as a function of wavelength. If the temperature is not high enough to dissociate all the molecules, such as $\mathrm{CO}$, they will remain everywhere in the atmosphere. Thus, the amplitude of their features in the transmission spectrum will be larger because they come from the hot regions of the inflated day-side atmosphere. On the other hand, the molecules that dissociate more easily, such as $\mathrm{H}_{2} \mathrm{O}$, will only remain on the cold night-side atmosphere. Therefore, the amplitude of the features of those molecules in the transmission spectrum will be less pronounced, 
since they come from colder regions on the night side of the atmosphere. A 1D retrieval code that tries to fit the spectrum with an isothermal atmosphere cannot unravel the information of a more complex temperature distribution, thus retrieving abundances that are biased. In particular, we demonstrate that for UHJs 1D retrieval would be biased toward high values of $[\mathrm{CO}] /\left[\mathrm{H}_{2} \mathrm{O}\right]$, the higher the ratio, the stronger the chemical heterogeneities. For instance, the TauREx retrieval code finds the planetary temperature according to the strength of the main spectral features. Since the amplitude of the spectral features is rather large because of the gas present on the hot day side of the planet, it is natural for a retrieval code to converge toward hotter temperatures. In the case of $\mathrm{H}_{2} \mathrm{O}$ dissociation, the only relevant gas present on the hot day side is $\mathrm{CO}$. The water absorption is due to the water present on the cold night side of the planet. Therefore, it is reasonable to retrieve a lower temperature in the case of water dissociation on the day side.

We demonstrated that a $3 \mathrm{D}$ atmospheric structure induces spectral distortions impossible to explain with a 1D retrieval. We also demonstrated that the water features can be strongly reduced by the presence of molecular heterogeneities due to a large day-night temperature contrast. For instance, we showed that a 3D geometry can explain some of the features observed in the HST/WFC3 wavelength range of Wasp-121b, even in the absence of hazes or clouds, thanks to the dissociation of water on the day side of the atmosphere. However, our works do not exclude the presence of clouds in UHJ atmospheres; the 3D effects are a complementary contribution to fill the water windows in the data. Thanks to our results, we think that Wasp-121b would be so a good target for future observations. We propose that 3D structural effects should already be considered when we study hot and ultra-hot Jupiters to avoid erroneous conclusions. Moreover, future space missions such as JWST (Beichman et al. 2014) and ARIEL (Tinetti et al. 2018) will probe a very wide range in wavelength (from 0.6 to $28 \mu \mathrm{m}$ for JWST), and then 3D effects will be even more evident in other parts of the exoplanetary spectra that have not been observed yet.

Finally, after the analysis of an exoplanetary atmosphere with a $1 \mathrm{D}$ retrieval tool, the $\tilde{\chi}^{2}$-test on a $1 \mathrm{D}$ retrieval can raise a warning about an important effect that the 1D model cannot consider, such as effects induced by the 3D structure of the exoplanetary atmosphere. In such cases, a parameterized 2D retrieval approach may be warranted. Whether there is enough information in the spectrum to actually constrain such a 2D approach remains to be demonstrated.

Acknowledgements. This project has received funding from the European Research Council (ERC) under the European Union's Horizon 2020 research and innovation programme (grant agreement $n^{\circ} 679030 /$ WHIPLASH).

\section{References}

Arcangeli, J., Désert, J.-M., Line, M. R., et al. 2018, ApJ, 855, L30 Arcangeli, J., Désert, J.-M., Parmentier, V., et al. 2019, A\&A, 625, A136 Barton, E. J., Yurchenko, S. N., \& Tennyson, J. 2013, MNRAS, 434, 1469 Barton, E. J., Chiu, C., Golpayegani, S., et al. 2014, MNRAS, 442, 1821 Beichman, C., Benneke, B., Knutson, H., et al. 2014, PASP, 126, 1134 Bell, T. J., \& Cowan, N. B. 2018, ApJ, 857, L20

Caldas, A., Leconte, J., Selsis, F., et al. 2019, A\&A, 623, A161

Changeat, Q., Edwards, B., Waldmann, I. P., \& Tinetti, G. 2019, ApJ, 886, 39

Cowan, N. B., Greene, T., Angerhausen, D., et al. 2015, PASP, 127, 311

Delrez, L., Santerne, A., Almenara, J.-M., et al. 2016, MNRAS, 458, 4025

Espinoza, N., Fortney, J. J., Miguel, Y., Thorngren, D., \& Murray-Clay, R. 2017, ApJ, 838, L9

Evans, T. M., Sing, D. K., Wakeford, H. R., et al. 2016, ApJ, 822, L4

Evans, T. M., Sing, D. K., Kataria, T., et al. 2017, Nature, 548, 58

Fu, Q., \& Liou, K. N. 1992, J. Atmos. Sci., 49, 2139

Goody, R. M., \& Yung, Y. L. 1989, Atmospheric Radiation : Theoretical Basis (Oxford: Oxford University Press)

Gordon, I. E., Kassi, S., Campargue, A., \& Toon, G. C. 2010, in 65th International Symposium On Molecular Spectroscopy, WF03

Gordon, I. E., Rothman, L. S., \& Li, G. 2013, in 68th International Symposium on Molecular Spectroscopy, ERE03

Greene, T. P., Line, M. R., Montero, C., et al. 2016, ApJ, 817, 17

Haynes, K., Mandell, A. M., Madhusudhan, N., et al. 2015, ApJ, 806, 146

Kataria, T., Showman, A. P., Lewis, N. K., et al. 2013, ApJ, 767, 76

Kataria, T., Showman, A. P., Fortney, J. J., et al. 2015, ApJ, 801, 86

Kreidberg, L., Line, M. R., Parmentier, V., et al. 2018, AJ, 156, 17

Lenzuni, P., Chernoff, D. F., \& Salpeter, E. E. 1991, ApJS, 76, 759

Lewis, N. K., Parmentier, V., Kataria, T., et al. 2017, ArXiv e-prints [arXiv:1706.00466]

Line, M. R., Wolf, A. S., Zhang, X., et al. 2013, ApJ, 775, 137

Lodders, K., \& Fegley, B. 2002, Icarus, 155, 393

Madhusudhan, N. 2012, ApJ, 758, 36

Marley, M. S., \& McKay, C. P. 1999, Icarus, 138, 268

Marley, M. S., Saumon, D., Fortney, J. J., et al. 2017, AAS Meeting Abstracts, 230, A 315.07

Oreshenko, M., Lavie, B., Grimm, S. L., et al. 2017, ApJ, 847, L3

Parmentier, V., Showman, A. P., \& Lian, Y. 2013, A\&A, 558, A91

Parmentier, V., Fortney, J. J., Showman, A. P., et al. 2016, ApJ, 828, 22

Parmentier, V., Line, M. R., Bean, J. L., et al. 2018, A\&A, 617, A110

Rothman, L. S., Gordon, I. E., Barbe, A., et al. 2009, J. Quant. Spectr. Rad. Transf., 110, 533

Sheppard, K. B., Mandell, A. M., Tamburo, P., et al. 2017, ApJ, 850, L32

Showman, A. P., Fortney, J. J., Lian, Y., et al. 2009, ApJ, 699, 564

Showman, A. P., Lewis, N. K., \& Fortney, J. J. 2015, ApJ, 801, 95

Stevenson, K. B., Lewis, N. K., Jacob L., Bean, et al. 2016, PASP, 128, 094401

Sudarsky, D., Burrows, A., \& Pinto, P. 2000, ApJ, 538, 885

Tan, X., \& Komacek, T. D. 2019, ApJ, 886, 26

Tennyson, J., \& Yurchenko, S. N. 2012, MNRAS, 425, 21

Tinetti, G., Drossart, P., Eccleston, P., et al. 2018, Exp. Astron., 46, 135

Visscher, C., Lodders, K., \& Fegley, Jr. B. 2006, ApJ, 648, 1181

Visscher, C., Lodders, K., \& Fegley, Jr. B. 2010, ApJ, 716, 1060

Waldmann, I. P., Rocchetto, M., Tinetti, G., et al. 2015a, ApJ, 813, 13

Waldmann, I. P., Tinetti, G., Rocchetto, M., et al. 2015b, ApJ, 802, 107

Wright, J. T., Marcy, G. W., Howard, A. W., et al. 2012, ApJ, 753, 160

Yurchenko, S. N., Barber, R. J., \& Tennyson, J. 2011, MNRAS, 413, 1828

Yurchenko, S. N., Tennyson, J., Bailey, J., et al. 2014, Proc. Natl. Acad. Sci., 111, 9379 